\title{
NANOPUBLICAÇÕES E INDEXAÇÃO: PROCESSOS E RELAÇÕES
}

\author{
NANOPUBLICACIONES E INDEXACIÓN: PROCESOS Y \\ RELACIONES
}

Lorena Tavares de Paula - Iorena.Itp@gmail.com Doutoranda em Ciências da Informação pela Universidade Federal de Minas Gerais (UFMG).

Maria Aparecida Moura - cidamoura@gmail.com Doutora em Comunicação e Semiótica pela Pontifícia Universidade Católica de São Paulo (PUC-SP). Professora da Universidade Federal de Minas Gerais (UFMG).

\section{RESUMO}

Introdução: O estudo parte da premissa de que indexar e disponibilizar conceitos na fonte primária pode oferecer subsídios ao compartilhamento e à colaboração científica na construção de novos conhecimentos. Apresenta-se o conceito de nanopublicações (nanopublications) e estabelece-se uma interconexão entre esse modelo emergente de representação da informação em ambientes digitais e as diretrizes postuladas pelo processo de indexação de informação.

Objetivo: Apresenta-se o conceito de nanopublicações em interface com a indexação, no âmbito dos processos de organização da informação em contexto. Realiza-se um experimento de modelagem de nanopublicações que teve por objetivo analisar a viabilidade de elaboração de uma metodologia de organização de informação que permita a criação um modelo de representação de informação em ambientes digitais para a difusão de conhecimentos em rede.

Metodologia: A metodologia foi estabelecida em duas etapas. Na primeira etapa, realiza-se o cotejamento conceitual relativo à temática nanopublicações e indexação. Na segunda etapa, baseada nas orientações da nanopublicação, estabelece-se a dimensão informacional do método através da geração de mapeamento dos elementos do corpus, da extração conceitual, da aplicação dos princípios de indexação e da modelagem da nanopublicação. Foram selecionados e analisados artigos relacionados ao conceito "netnografia" a partir de seleção realizada no "Google Scholar". Os artigos obtidos 
pertenciam a um campo comum de conhecimento, a Ciência Social Aplicada, e a domínios distintos como: Comunicação Social, Administração e Ciência da Informação.

Resultado: A elaboração da nanopublicação sobre "netnografia" proporcionou a percepção de que a interlocução metodológica com os princípios de indexação é possível e eficiente. O experimento proposto neste artigo corroborou o propósito da atribuição de crédito às citações, identificadas e caracterizadas na nanopublicação, pois além da identificação das principais definições de "netnografia" foi evidenciado o autor deste conceito, o qual foi citado em todos os artigos analisados e mencionado como a principal referência nos estudos netnográficos. Além disso, ficou expressa a necessidade de representação temática de dois elementos no corpo de metadados, segundo as e-evidências. O primeiro está relacionado à citação direta em nanopublicações e o segundo está orientado à identificação das comunidades de origem dos autores dos artigos.

Conclusões: Considera-se que, diante dos visíveis avanços do conhecimento e da consequente explosão informacional em rede, devem-se buscar maneiras de impulsionar o uso da crescente produção documental, através de mecanismo de representação de informações coerentes com as demandas sócio/tecnológicas vigentes. $\mathrm{Na}$ atualidade, produzir conhecimento de maneira ágil, exige formas de recuperação de informação tão precisas quanto às unidades citáveis caracterizadas pelas nanopublicações.

Palavras-chave: Nanopublicação. Indexação. Organização da informação em contexto metodologia.

\section{INTRODUÇÃO}

Os ambientes digitais, baseados em tecnologias de Internet, objetivam a fluidez da informação de modo a proporcionar aos seus usuários interação eficiente com o espaço documental acessado. Nestes ambientes as técnicas e tecnologias orientadas à organização da informação buscam promover uma melhor relação entre usuários e conteúdos, considerando as possibilidades da infraestrutura digital e suas alternativas para a colaboração e disseminação informacional.

No que diz respeito a esse contexto identifica-se autores como Moura (2009), que analisam a informação como um signo que se atualiza na interface com o sujeito, "algo que se forma sem cessar". De acordo com a autora,

[...] se antes as ações teóricas e experimentos práticos tinham como foco o aspecto material da informação, hoje, devido aos intercâmbios entre os papéis sociais de autores e receptores de informação, é possível afirmar que o foco migrou para a recuperação de trechos informacionais. $\mathrm{E}$ devido à contigüidade presente nos acervos digitalmente dispostos em rede, o usuário de informação está interessado em encontrar um trecho, ou um segmento de um documento, e não necessariamente, um documento em sua manifestação física (MOURA, 2009, p. 32). 
A afirmativa apresentada por Moura (2009) faz uma referência crítica aos processos clássicos de indexação de conteúdo frente as novas demandas dos usuários em ambientes digitais em rede, pois o acesso ao documento completo por meio de descritores já não é considerado o ideal de precisão informacional. Naqueles processos de indexação ocorre a representação da informação com termos que correspondem simultaneamente ao conteúdo intelectual do documento e a provável intenção de busca do usuário para acionar um conteúdo. Entretanto, diante da abundância informacional publicada em meio digital, pode se fazer necessária a seleção de fragmentos de textos específicos.

Com o objetivo de elaborar e prover acesso a fragmentos de textos contextualizados em publicações digitais, desenvolveu-se as nanopublicações, descritas como "a menor unidade de informação publicável: é caracterizada como uma extração textual baseada exclusivamente na 'fala do autor'". (NANOPUB.ORG, online).

O presente artigo pretende apresentar e discutir as nanopublicações e estabelecer uma interface desse modelo emergente de representação da informação com as diretrizes postuladas pelo processo de indexação de informação.

Com esse propósito será apresentado a gênese das nanopublicações para, posteriormente, estabelecer uma descrição dos processos de indexação que podem interferir e orientar a sua construção. A partir dos entendimentos conceituais será estabelecido um breve experimento metodológico, utilizando 6 (seis) artigos que tratam do assunto "netnografia", para demonstrar o uso de mecanismos de indexação para a construção de nanopublicações. Salienta-se que as camadas tecnológicas que se referem às nanopublicações não foram abordadas. O que se apresenta neste artigo é uma interlocução entre a modelagem de nanopublicações e os princípios de indexação. Por fim, serão tecidas considerações sobre métodos e conceitos que podem auxiliar na consolidação desse modelo de representação emergente.

\section{NANOPUBLICAÇÃO: GÊNESE E HISTÓRICO}

A nanopublicação, como já foi explicitada, pode ser considerada a menor unidade de informação publicada em um documento formal: ela é uma extração textual atribuída a "fala do autor". Segundo a Web Alliance Concept (CWA) as 
nanopublicações podem ser citadas, pois são uma referência exata das ideias contidas em uma publicação formal. Elas possibilitam uma comunicação que se baseia em informações contextuais de alta qualidade e que servem para a divulgação, apropriação e organização de informações contextualizadas de acordo com um autor e uma unidade conceitual.

Groth, Gibson e Velterop (2011) explicam que a elaboração de uma nanopublicação requer conteúdos estruturais que reforcem 0 valor de uma declaração ao ponto em que ela possa ser considerada uma publicação. A Web Alliance Concept (CWA), em seu compromisso de permitir um diálogo aberto e colaborativo, para enfrentar os desafios associados com alto volume de dados acadêmicos, explica que o surgimento das nanopublicações foi observado em 2009 em Nova York no encontro dos "Setenta mais" $(70+)$ especialistas em dados e tecnologias de web semântica a Web Alliance Concept(CWA) (NAONPUB.ORG, 2014).

No mesmo ano, Barend Mons e Jan Velterop apresentaram as "nanopublications" no artigo "Nanopublication in the e-science" no W3C HCLS (2009), workshop sobre aplicações da web na semântica no discurso científico. Em Janeiro de 2010 Paul Groth, Andrew Gibson, e Jan Velterop delinearam especificações técnicas iniciais para as nanopublicações no artigo "The Anatomy of a Nanopublication". Posteriormente, em 2011, observa-se o trabalho "The Open Pharmaceutical' (open PHACTS), projeto que adota nanopublicações para auxiliar no acesso objetivo de dados. Este trabalho é a primeira implementação de uma estrutura de nanopublicação (NAONPUB.ORG, 2014).

Em Abril de 2011 efetiva-se uma ação fundamental para a consolidação do conceito de nanopublicação quando o Nature Genetics publica um editorial e um artigo de investigação sobre micro-allocation e nanopublications. Neste mesmo ano o site nanopub.org vai ao ar. No ano seguinte em 2012 a open PHACTS libera suas primeiras "Diretrizes de Nanopublicação" e lança a primeira versão da plataforma "Espaço farmacológico aberto" para testes internos "Alpha".

Segundo o Naonpub.org após a constatação de que o projeto open phacts é um esforço limitado de tempo, a CWA inicia atividades para se tornar uma organização executiva, tendo como um de seus principais objetivos hospedar versões progressivas das orientações de nanopublicações, a fim de ajudar na transformação de dados brutos em dados interoperáveis (NAONPUB.ORG, 2014). 
Groth, Gibson e Velterop (2011) explicam que, em seu aspecto conceitual as nanopublicações devem ser citáveis, atribuíveis e revisáveis. Além disso, elas precisam ser reorganizáveis sem dificuldades, pois "as nanopublicações devem ser facilmente agregadas e identificadas em toda a web". Elas precisam ser extensíveis para atender as novas formas de descrição orientadas por conjuntos de definições como:

1. Definição Conceitual: conceito entendido como a menor unidade, livre de ambiguidades;

2. Sintaxe: envolvimento de três conceitos (sujeito, predicado, objeto);

3. Declaração - Uma sintaxe que é exclusivamente identificável;

4. Anotação - Uma declaração sobre a sintaxe que reforce o assunto e seja referenciável;

Groth, Gibson e Velterop (2011) esclarecem que a nanopublicação pode ser considerada um conjunto de anotações que se referem a declarações e conteúdos orientados a comunidades que propõem as definições conceituais. A partir disso, ela pode atender a alguns requisitos básicos como:

- A capacidade de identificação conceitual;

- A capacidade de identificação de comunidades;

- Capacidade de compilação de conceitos e declarações;

- Em uma perspectiva computacional, permitir interoperabilidade com diferentes formatos computacionais.

Além disso, diante da sua configuração computacional ocorrem também os requisitos:

1 fromSource (identifica onde a declaração de pesquisa foi extraída);

2 importedBy (identifica que a entidade é responsável pela importação da declaração);

3 authoredBy (identifica o autor de uma declaração de pesquisa).

Observa-se que essa modalidade de publicação faculta aos autores, editores e pesquisadores a possibilidade de disponibilizarem seus conteúdos em formatos que impulsionam o acesso de suas produções de acordo com o que consideram mais relevantes para o impacto sócio/comunicacional de sua obra. Deste modo, as 
Nanopublicações apresentam dois elementos básicos:

1. A afirmação: Uma afirmação é uma unidade mínima do pensamento, que expressa uma relação entre dois elementos (chamados o sujeito e o objeto) usando um terceiro elemento (chamado de predicado).

2. A Proveniência: Este elemento fornece o contexto sobre a afirmação. Proveniência significa, "como isso veio a ser" e pode incluir a descrição do método e algumas atribuições (como autores, instituições, tempo , subvenções, links internos e externos, URLs).

Ressalta-se que as nanopublicações podem ser realizadas usando ontologias existentes e RDF (Resource Description Framework) e permitem a leitura, em processo de interoperabilidade. Além disso, há a possibilidade de compatibilizar informações heterogêneas e descentralizadas, contribuindo para a descoberta de novas associações, que auxiliam na construção de novos conhecimentos.

\section{$3 \quad$ PROCESSOS DE INDEXAÇÃO}

O processo de indexação obedece a dois princípios básicos:

1 - Análise Conceitual;

2 - Tradução para uma linguagem de indexação.

Lancaster (2004) explica que a indexação e o resumo são "atividades intimamente relacionadas, pois ambas implicam a preparação de uma representação do conteúdo temático dos documentos". O indexador emprega vários termos de indexação que possuem relação com o conteúdo intelectual do documento e a provável indagação de busca de informação do usuário a fim de estabelecer uma interação eficiente entre sujeito e mecanismo de recuperação de informação.

No primeiro momento da indexação, quando em um processo de análise intelectual são extraídos conceitos, ocorre a análise de assuntos que remetem ao conteúdo intelectual tratado na publicação. Neste mesmo momento, pode ocorrer uma exploração conceitual na interconexão de familiaridades de questões como as sugeridas por Wilson (1968). Os processos sugeridos por este autor estão ligados ao exame de tabelas de títulos, do índice, da introdução, dos resumos, etc. Mas há também questionamentos que podem revelar conteúdos pertinentes com as 
necessidades do usuário, como: O que quer dizer? Pra quem quer dizer? O que se está fazendo entender? São interrogações plausíveis para a identificação do assunto, pois o que 0 autor quer transmitir está condicionado a noções prévias de seus leitores, que estabelecem articulações de acordo com sua enciclopédia sócio/cultural.

$\mathrm{Se}$ as perguntas fossem orientadas ao autor, ou editor, poder-se-ia obter respostas como: - Qual assunto você está escrevendo? O que quer dizer isto? Qual sua principal abordagem? Dessa forma se chegaria a uma definição "quase exata" do que o documento quer realmente comunicar. Entretanto, uma das reflexões sobre a impossibilidade de descrição exata de um assunto escrito é confirmada por Wilson (1968). Ele explica que um documento retrata a aparência da realidade de um dado contexto. Suas leituras são plausíveis de interpretações diversas, mas existem itens que são comuns a todos que o leiam.

A análise conceitual implica decidir do que trata o documento. Uma indexação de assunto eficiente implica que se tome uma decisão não somente quanto ao que é tratado no documento, mas também por que ele se reveste de provável interesse para determinado grupo de usuários.

O processo de análise pode observar as seguintes estruturas no processo de indexação. 


\section{Quadro 1 - Descrição do Registro}
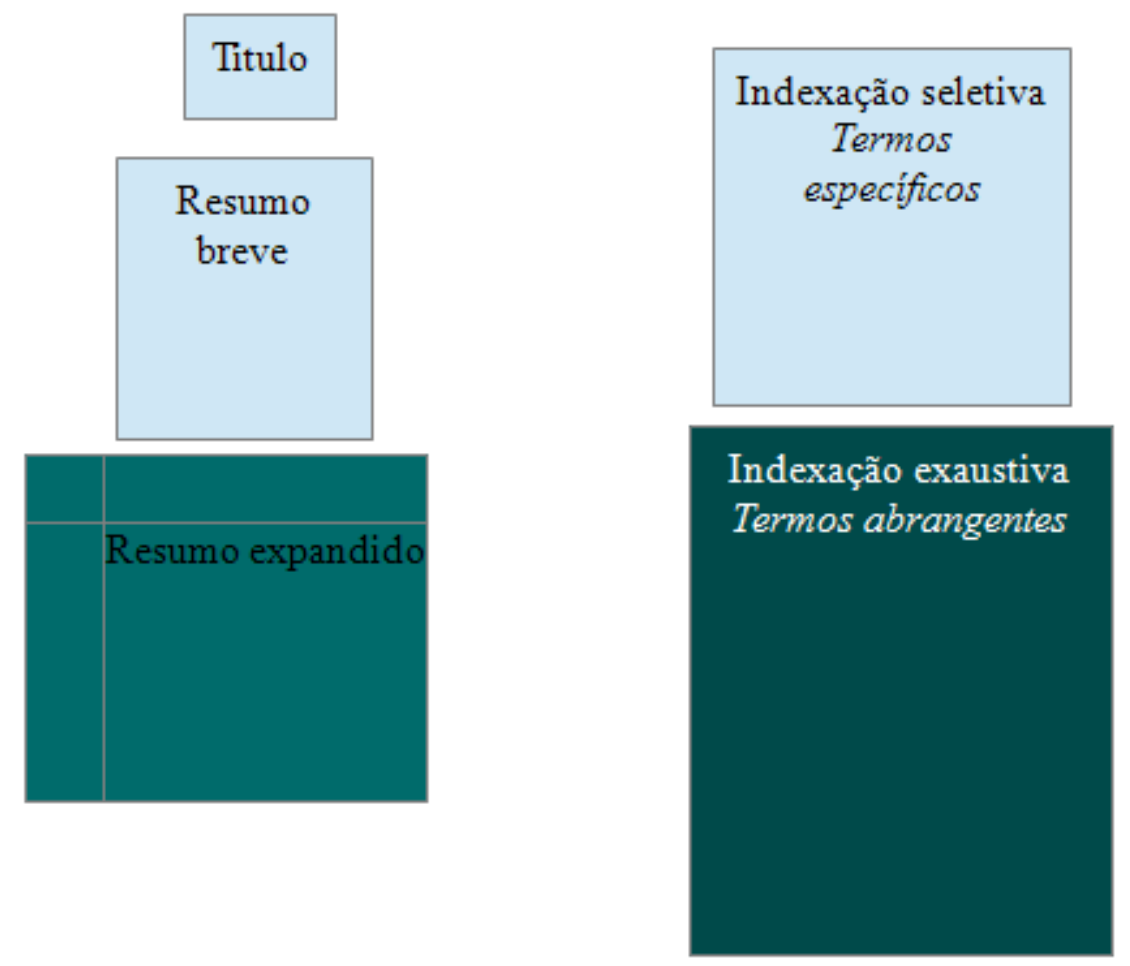

Fonte: Adaptado de Lancaster (2004).

O Quadro 1 é um modelo baseado no "efeito do registro sobre recuperabilidade" de Lancaster (2004), percebe-se que quanto mais informações são apresentadas, mais claramente a representação revela o alcance do documento. Deste modo, o leitor poderá ter o máximo de certeza se essa informação satisfaz ou não suas necessidades. A princípio observa-se o "título", que pode não oferecer especificidade sobre os tópicos tratados no documento. No "resumo breve", pode-se obter informações focalizadas no tópico central da publicação. Já o "resumo ampliado" oferece conteúdos abrangentes que correspondem às várias seções da publicação, bem como algumas de suas intenções. A "indexação exaustiva" ira incluir termos que indicam de maneira geral todos os assuntos plausíveis de indexação no documento e a "indexação seletiva" proporcionará uma indicação minuciosa dos assuntos específicos e relevantes.

Lancaster (2004) destaca que quanto mais especializada a clientela de um centro de informação maior a probabilidade de que a indexação possa e deva ser feita sob medida, ajustando-se com precisão aos interesses do grupo.

Hjørland (1997, p. 776) acrescenta que: 


\begin{abstract}
Uma vez que qualquer documento pode, em princípio, proporcionar respostas a uma infinidade de questões, as análises de assunto devem estabelecer propriedades baseadas nos grupos de usuários específicos atendidos. $\mathrm{O}$ assunto de um documento é assim relativo ao objetivo do serviço de informação específico. Defino assunto [...] como potenciais epistemológicos ou informativos dos documentos. A melhor análise temática é a que faz o melhor prognóstico quanto ao uso futuro do documento.
\end{abstract}

Todo o processo de indexação objetiva exclusivamente a recuperação da informação, a qual Cesarino e Pinto (1985) descrevem como um "conjunto de cooperações consecutivas executadas para localizar, dentro da totalidade de informações disponíveis, aquelas realmente relevantes". A partir da descrição de assunto em forma de termos descritores pode-se promover uma integração entre documento, acervo e usuário.

Salienta-se que a análise de assunto é a chave para toda a indexação efetiva. Neste processo, é sempre importante analisar o documento em suas minúcias de seções de capítulos no qual se pode estabelecer uma análise tão exaustiva quanto a relevância do documento para a comunidade de usuários da unidade de informação. Neste sentido, a indexação e as nanopublicações convergem com a necessidade de identificação de conteúdos que ofereçam especificidade ao documento no momento da recuperação da informação.

\title{
4 NANOPUBLICAÇÃO E INDEXAÇÃO: SUGESTÃO METODOLÓGICA
}

Nesta seção será proposto um experimento com o uso da indexação para a construção de uma nanopublicação. Ressalta-se que o objetivo desse experimento é evidenciar uma possível interlocução entre os dois processos a fim de expor uma metodologia híbrida para processos de extração textual citável.

Inicialmente serão delimitados 2 (dois) processos analíticos para extração e assimilação de informações de publicações, o primeiro baseado nas premissas da indexação e o segundo relacionado às junções: sujeito, predicado e objeto, característicos das nanopublicações. 
Figura 1 - Processo de extração de conceitos

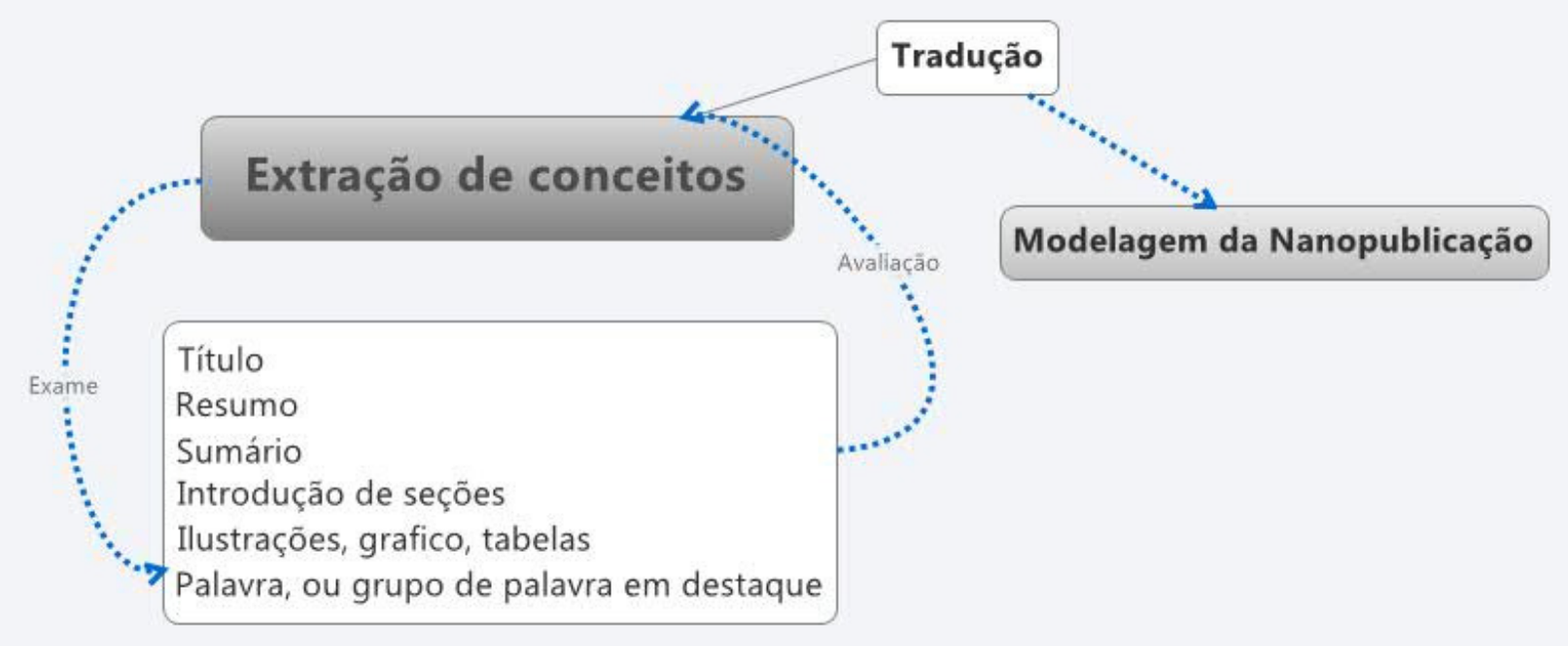

Fonte: Elaborado pelas autoras, baseando-se em Lancaster (2004).

Na figura 1 percebe-se os principais elementos textuais, nos quais a extração de conceitos pode ser efetivada: título, resumo sumário, introduções de seções, ilustrações e palavras em evidência. O destaque para esses elementos não quer dizer que a extração de conceito deva se limitar a eles, mas que esses são espaços em que normalmente se encontram os insumos necessários para identificação e/ou localização de definições conceituais relevantes. Além disso, o conhecimento prévio sobre o domínio no qual se realiza a extração de conceitos faz-se imprescindível para uma verificação crítica das escolhas estabelecidas. Durante a fase de extração conceitual não há necessidade de consulta a um vocabulário controlado, esse exercício de tradução será pensado na etapa de elaboração da nanopublicação.

Feita a identificação de ocorrência do conceito central a ser extraído, faz-se em sequência um exame da definição conceitual estabelecida, para posteriormente estabelecer uma avaliação das definições conceituais que irão compor a nanopublicação, momento em que serão estabelecidas relações entre os termos, a fim de compor as declarações e anotações que legitimam essa construção representacional. 
Figura 2 - Modelagem da nanopublicação

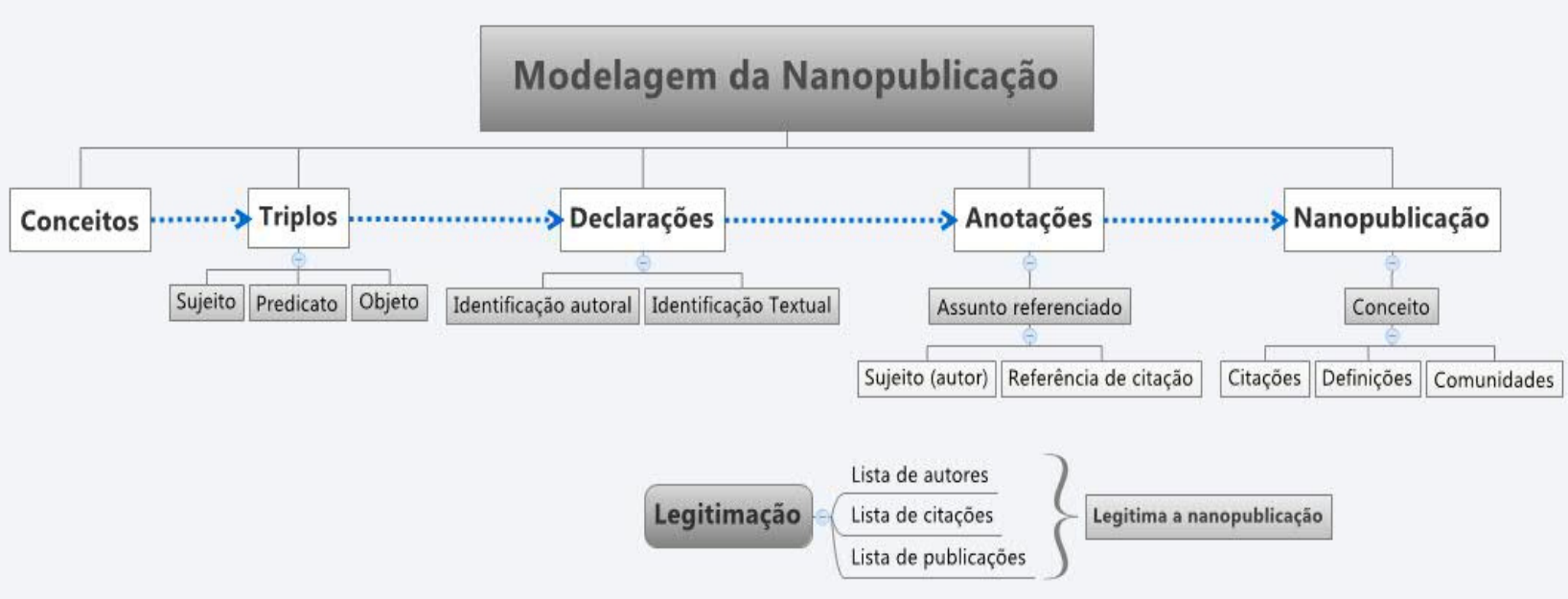

Fonte: Elaborado pelas autoras.

A figura 2 apresenta o processo de modelagem de uma nanopublicação. De início são estabelecidos os conceitos, que foram retirados durante a fase de análise de assunto baseada nos princípios de indexação. Estes conceitos são considerados a menor unidade, sem ambiguidades que expressa um pensamento exclusivo e identificável. Posteriormente, são ajustados os processos de triplo (sintaxe), no qual são estabelecidas as relações entre: sujeito, predicado e objeto, neste momento ocorre a relação de ação (causa/efeito) sobre o conceito e inicia-se a elaboração da nanopublicação.

A construção da declaração é estabelecida a partir das relações entre os termos extraídos, quando pode-se elaborar um mapa conceitual, e a identificação das definições extraídas dos artigos com suas devidas identificações de autoria. As anotações veem em sequência, para oferecer uma declaração que abranja o assunto estabelecido ao longo da modelagem. Por fim, a nanopublicação é estabelecida como um conjunto de anotações que consolidam declarações de publicações oriundas de comunidades distintas. Além disso, são estabelecidas as eevidências, que são a caracterização da Nanopublicação evidenciada em uma sugestão de metadados, para a representação do "conceito" como "menor unidade citável" e proporcionar confirmações bibliográficas de todas as afirmações ajustadas na nanopublicação. Em resumo, no momento da especificação das e-evidências são acordados os metadados apropriados para a identificação da nanopublicação.

A partir da observação do processo metodológico, foi estabelecido um breve experimento composto por 6 (seis) publicações da área de Ciências Sociais 
Aplicadas, todos em língua portuguesa. Essas publicações possuem como assunto comum a "Netnografia" e o objetivo da análise desses artigos é promover uma nanopublicação sobre a definição de "Netnografia".

\section{EXPERIMENTO: MODELAGEM DO CONCEITO DE "NETNOGRAFIA"}

Os artigos selecionados para o experimento estão descritos na tabela abaixo.

Tabela 1 - Artigos selecionados para o experimento

\begin{tabular}{|ll|}
\hline Títulos artigos de netnografia & Autores \\
\hline $\begin{array}{l}\text { Estudo dos blogs a partir da netnografia: } \\
\text { possibilidades e limitações }\end{array}$ & $\begin{array}{l}\text { Sandra Portella Montardo } \\
\text { Liliana Maria Passerino }\end{array}$ \\
\hline $\begin{array}{l}\text { Netnografia como aporte metodológico da } \\
\text { pesquisa em comunicação digital* }\end{array}$ & $\begin{array}{l}\text { Adriana Amaral } \\
\text { Geórgia Natal } \\
\text { Lucina Viana }\end{array}$ \\
\hline $\begin{array}{l}\text { Perspectivas do método etnográfico em } \\
\text { marketing: consumo, comunicação e } \\
\text { netnografia }\end{array}$ & $\begin{array}{l}\text { Everardo Pereira } \\
\text { Quimarães Rocha Carla Barros } \\
\text { Claudia Pereira }\end{array}$ \\
\hline $\begin{array}{l}\text { Netnografia: incursões metodológicas na } \\
\text { cibercultura }\end{array}$ & $\begin{array}{l}\text { Paula Jung Rocha, } \\
\text { Sandra Portella Montardo }\end{array}$ \\
\hline $\begin{array}{l}\text { Netnografia": uma abordagem para estudos } \\
\text { de usuários no ciberespaço }\end{array}$ & $\begin{array}{l}\text { Virginia Pinto, } \\
\text { Casimiro Neto, } \\
\text { Maria Fátima Costa, } \\
\text { Fabiola Bezerra, } \\
\text { Heliomar Sobrinho, } \\
\text { Maria Fátima Cysne }\end{array}$ \\
\hline $\begin{array}{l}\text { Apontamentos metodológicos iniciais sobre a } \\
\text { netnografia no contexto pesquisa em } \\
\text { comunicação digital e cibercultura. }\end{array}$ & $\begin{array}{l}\text { Adriana Amaral; } \\
\text { Geórgia Natal; } \\
\text { Lucina Viana }\end{array}$ \\
\hline
\end{tabular}

Fonte: Elaborado pelas autoras.

A tabela 1 apresenta os artigos e suas respectivas autorias. A seleção desses artigos obedeceu ao critério de pesquisa "netnografia" no "Google Scholar". Nesta busca foi obtido um resultado de 738 itens nos quais constavam cerca de 10 páginas sequenciais com 10 itens cada. Foi feita a opção de seleção dos artigos da primeira e segunda página de resultados do google scholar $^{1}$, pois essas publicações pertenciam a um campo comum de conhecimento que é a Ciência Social Aplicada e a domínios distintos como: Comunicação Social, Administração e Ciência da Informação.

1 Pesquisa Google Scholar: http://scholar.google.com.br/scholar?hl=pt-BR\&q=netnografia\&btnG=\&lr= 
A partir da separação dos artigos iniciou-se a primeira fase do experimento que trata da extração conceitual de acordo com as orientações dos princípios de indexação descritos na metodologia. Neste processo obteve-se a seguinte tabela:

Tabela 2 - Extração conceitual

\begin{tabular}{|c|c|c|c|c|c|}
\hline Artigos & Títulos & $\begin{array}{l}\text { Aréa de } \\
\text { conhecimento }\end{array}$ & Titulo & \begin{tabular}{||l||l} 
& Conceitos \\
Palavra-Chave & Extraídos do resumo
\end{tabular} & \multirow{7}{*}{$\begin{array}{l}\text { Netnografia } \\
\text { Cibercultura } \\
\text { Metodologia } \\
\text { Etnografia } \\
\text { Comunicação } \\
\text { Comunidades } \\
\text { online }\end{array}$} \\
\hline 1 & $\begin{array}{l}\text { Estudo dos blogs a partir da } \\
\text { netnografia: possibilidades } \\
\text { limitações }\end{array}$ & Comunicação social & $\begin{array}{l}\text { Blog } \\
\text { Netnografia }\end{array}$ & $\begin{array}{lll} & \text { Blogosfera } \\
& \text { Blog } \\
& \text { Internet } \\
\text { blogs; netnografia; } & \text { metodologia } \\
\text { metodologia } & \text { de Etnografia } \\
\text { pesquisa } & \text { Netnografia }\end{array}$ & \\
\hline 2 & $\begin{array}{l}\text { Netnografia como aporte } \\
\text { metodológico da pesquisa } \mathrm{em} \\
\text { comunicação digital* }\end{array}$ & Comunicação Social & $\begin{array}{l}\text { Netnografia } \\
\text { Comunicação } \\
\text { digital }\end{array}$ & $\begin{array}{lc}\text { Netnografia } & - \\
\text { Metodologia } & \text { de Netnografia } \\
\text { pesquisa } & \text { - Metodologia } \\
\text { Cibercultur } & \text { comunidades online }\end{array}$ & \\
\hline 3 & $\begin{array}{l}\text { Perspectivas do método etnográfico } \\
\text { em marketing: consumo, comunicação } \\
\text { e netnografia }\end{array}$ & Administração & $\begin{array}{l}\text { Etnografia } \\
\text { Consumo } \\
\text { Comunicação } \\
\text { Netnografia }\end{array}$ & $\begin{array}{l}\text { Etnografia } \\
\text { Metodologia } \\
\text { Marketing } \\
\text { Netnografia } \\
\text { Possitivismo }\end{array}$ & \\
\hline 4 & $\begin{array}{l}\text { Netnografia: incursões metodológicas } \\
\text { na cibercultura }\end{array}$ & Comunicação Social & $\begin{array}{l}\text { Netnografia } \\
\text { Metodologia } \\
\text { Cibercultura }\end{array}$ & $\begin{array}{ll}\text { Cibercultura, } & \\
\text { comunicação, } & \text { Netnografia } \\
\text { antropologia, } & \text { Ciberespaço } \\
\text { metodologia } & \text { e Comunicação } \\
\text { etnografia } & \text { Não lugar }\end{array}$ & \\
\hline 5 & $\begin{array}{l}\text { Netnografia": uma abordagem para } \\
\text { estudos de usuários no ciberespaço }\end{array}$ & $\begin{array}{l}\text { Ciência } \\
\text { Informação }\end{array}$ & $\begin{array}{l}\text { Netnografia } \\
\text { Ciberespaço }\end{array}$ & 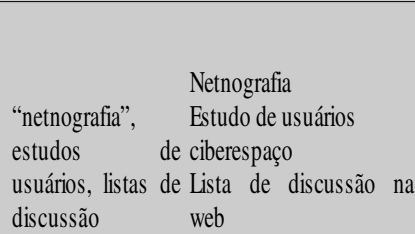 & \\
\hline 6 & $\begin{array}{l}\text { Apontamentos metodológicos iniciais } \\
\text { sobre a netnografia no contexto } \\
\text { pesquisa em comunicação digital e } \\
\text { cibercultura. }\end{array}$ & Ciências Sociais & $\begin{array}{l}\text { Netnografia } \\
\text { Metodologia } \\
\text { Comunicação } \\
\text { Digitalmente } \\
\text { Cibercultura }\end{array}$ & $\begin{array}{l}\text { netnografia; } \\
\text { metodologia de } \\
\text { pesquisa; } \quad \text { Netnografia } \\
\text { comunicação } \quad \text { Metodologia } \\
\text { digital; cibercultura }\end{array}$ & \\
\hline
\end{tabular}

Fonte: Elaborado pelas autoras.

A tabela 2 apresenta os conceitos extraídos dos artigos a partir do título, palavras chaves e resumo. Eles são normalmente coincidentes nos artigos analisados, entretanto há uma pequena variação de acordo com a temática apresentada nos conteúdos específicos de cada artigo. A publicação 1 (um) trata de um estudo de blogs desenvolvido no campo da comunicação social. O artigo 2 (dois) busca elucidar o uso da "netnografia" como estratégia para as pesquisas em comunicação social. Já o artigo 3 (três) pertence a área de administração, especificamente do marketing e objetiva observar a "netnografia" como método para 
estudo do consumo. Retomando a Comunicação Social, observa-se o artigo 4 (quatro) propondo uma incursão na cibercultura, verificando os sujeitos de comunidades digitais como sujeitos de um "não lugar". Em outra perspectiva a "netnografia" é implementada para estudos de usuários no campo de Ciência da Informação no artigo 5 (cinco). Por fim a pesquisa "Netnográfica" de comunidades digitais é analisada no artigo 6 (seis), especificamente em um estudo sociológico.

Diante da observação do processo de extração conceitual, feita a partir da análise dos artigos, chega-se a um desenho que demonstra todos os termos representativos, que estabelecerão as relações de ação (sujeito, predicado, objeto) na nanopublicação.

Figura 3 - Conceitos comuns

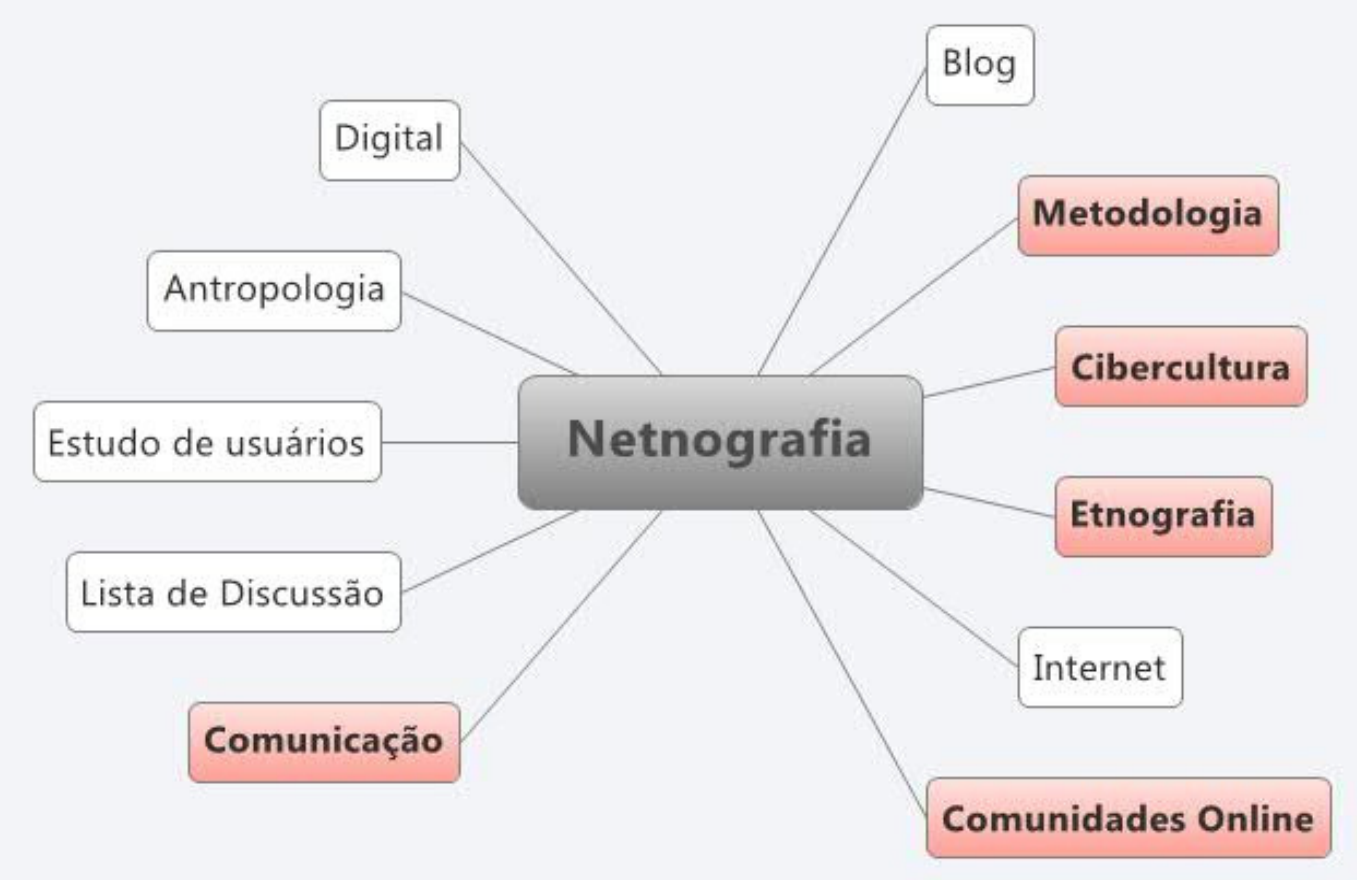

Fonte: Elaborado pelas autoras.

$\mathrm{Na}$ Figura 3 verifica-se que os conceitos de Etnografia, Metodologia, Comunicação, Cibercultura e Comunidades Online estão em destaque pela sua recorrente evidência nos artigos selecionados. Diante disso, as etapas de construção da nanopublicação foi estabelecida tendo como insumo os termos destacados.

Durante o processo de análise fez-se a opção de descrição (declaração e anotação) do assunto "netnografia" baseado-se em quatro artigos, dentre os seis 
selecionados. Esses artigos e suas respectivas descrições conceituais são apresentados na tabela 3. A opção por extrair fragmentos textuais de apenas quatro artigos fez-se pela constante coincidências na definição de "Netnografia", além da observação de temas e abordagens coincidentes nos artigos selecionados.

Tabela 3 - Extração textual

\begin{tabular}{|c|c|c|c|}
\hline \multicolumn{4}{|c|}{ Estudo dos blogs a partir da netnografia: possibilidades e limitações } \\
\hline Sujeito & Predicado & Objeto & Contextos de Citações \\
\hline Netnografia & estuda & $\begin{array}{l}\text { Comunidades } \\
\text { online }\end{array}$ & $\begin{array}{l}\text { "Segundo Kozinets (1997), a netnografia pode ser } \\
\text { empregada de três formas: (1) como ferramenta } \\
\text { metodológica para estudar comunidades virtuais puras; } \\
\text { (2) como ferramenta metodológica para estudar } \\
\text { comunidades virtuais derivadas; e (3) como ferramenta } \\
\text { exploratória para diversos assuntos". }\end{array}$ \\
\hline Netnografia & $\begin{array}{l}\text { observa } \\
\text { comunidades } \\
\text { na }\end{array}$ & Cibercultura & $\begin{array}{l}\text { "Para Kozinets (1997), a netnografia exige combinação } \\
\text { imersiva entre participação e observação cultural com } \\
\text { relação às comunidades pesquisadas, sendo que o } \\
\text { pesquisador deve ser reconhecido como um membro } \\
\text { da cultura, um elemento importante do trabalho de } \\
\text { campo" }\end{array}$ \\
\hline Netnografia & $\begin{array}{l}\text { deve ser } \\
\text { usada como } \\
\text { ferramenta } \\
\text { complementar } \\
\text { da }\end{array}$ & Metodologia & $\begin{array}{l}\text { "a netnografia deve ser utilizada como ferramenta } \\
\text { complementar a outros tipos de abordagem, como } \\
\text { entrevistas pessoais ou por telefone e grupos de } \\
\text { discussão" }\end{array}$ \\
\hline Netnografia & $\begin{array}{l}\text { permite a } \\
\text { exploração }\end{array}$ & Comunicação & $\begin{array}{l}\text { "A netnografia aplicada ao estudo dos blogs apresenta } \\
\text { como possibilidades a exploração da comunicação } \\
\text { multimídia, permitindo, contar com dados coletados em } \\
\text { texto, áudio e vídeo, recursos que podem enriquecer a } \\
\text { observação dos estudos etnográficos tradicionais" }\end{array}$ \\
\hline Netnografia & $\begin{array}{l}\text { é uma } \\
\text { adaptação }\end{array}$ & Etnografia & $\begin{array}{l}\text { "adaptações requeridas pela aplicação da técnica } \\
\text { etnográfica no ambiente da web foram inicialmente } \\
\text { introduzidos por Kozinets }(1997,2002) \text { e } \\
\text { posteriormente por Hine }(2005) . " \\
\text { "netnografia é uma adaptação da etnografia no } \\
\text { ambiente web" } \\
\text { "comunicação analisada em netnografia é diferente da } \\
\text { observada na etnografia tradicional” }\end{array}$ \\
\hline \multicolumn{4}{|c|}{ Estudo dos blogs a partir da netnografia: possibilidades e limitações } \\
\hline Sujeito & Predicado & Objeto & Contextos de Citações \\
\hline Netnografia & $\begin{array}{l}\text { aplicado a } \\
\text { redes sociais }\end{array}$ & $\begin{array}{l}\text { Comunidades } \\
\text { digitais }\end{array}$ & $\begin{array}{l}\text { "Simone Pereira de Sá, cujo texto Netnografias nas } \\
\text { redes digitais" }\end{array}$ \\
\hline Netnografia & aplicada à & Cibercultura & $\begin{array}{l}\text { "a netnografia vem sendo aplicada com maior } \\
\text { frequência e que no mundo acadêmico se observa, } \\
\text { particularmente, em pesquisadores de língua inglesa, } \\
\text { uma preocupação quanto à aplicação dos métodos da } \\
\text { etnografia virtual". }\end{array}$ \\
\hline Netnografia & ferramenta & Metodologia & Não foi identificada citação direta. \\
\hline
\end{tabular}




\begin{tabular}{|l|l|l|l|} 
& para à & & \\
\hline Netnografia & aplicada à & Comunicação & Não foi identificada citação direta \\
\hline Netnografia & $\begin{array}{l}\text { derivado da } \\
\text { técnica }\end{array}$ & Etnografia & $\begin{array}{l}\text { "Segundo Kozinets4 (2002) a netnografia é definida } \\
\text { como um método de pesquisa derivado da técnica } \\
\text { etnográfica desenvolvida no campo da antropologia e, } \\
\text { costuma-se dizer que a netnografia tem conhecido um } \\
\text { crescimento considerado devido à complexidade das } \\
\text { experiências da sociedade digital. Este método é } \\
\text { constantemente utilizado por pesquisadores das áreas } \\
\text { da comunicação, do marketing, da antropologia e da } \\
\text { sociologia" }\end{array}$ \\
\hline
\end{tabular}

"Netnografia": uma abordagem para estudos de usuários no ciberespaço

\begin{tabular}{|c|c|c|c|}
\hline Sujeito & Predicado & Objeto & Contextos de Citações \\
\hline Netnografia & \begin{tabular}{|l|} 
estudo \\
naturalista de
\end{tabular} & $\begin{array}{l}\text { Comunidades } \\
\text { Virtuais }\end{array}$ & $\begin{array}{l}\text { "Sherry e Kozinets (2000) a netnografia diz respeito a } \\
\text { fusão de técnicas etnográficas estabelecidas e } \\
\text { inovadoras, adaptadas ao estudo naturalista de } \\
\text { comunidades virtuais, e a sua reapresentação da } \\
\text { pesquisa, procurando obter experiências profundas da } \\
\text { sociabilidade digital" } \\
\\
\text { "o uso da netnografia para estudos de usurários de } \\
\text { bibliotecas digitais sugere várias possibilidades de } \\
\text { análise o que pode enriquecer as observações das } \\
\text { pesquisas de comportamentos de comunidades no } \\
\text { território Ciberespaciano". }\end{array}$ \\
\hline Netnografia & $\begin{array}{l}\text { abordagem } \\
\text { etnografica no }\end{array}$ & Cibercultura & $\begin{array}{l}\text { "netnografia, enquanto nova abordagem da etnografia } \\
\text { no ciberespaço, é possível conhecer os hábitos de } \\
\text { grupos de usuários desta lista uma vez que nela eles } \\
\text { podem navegar com maior liberdade e atribuir sentidos } \\
\text { aos produtos e serviços a eles ofertados" }\end{array}$ \\
\hline Netnografia & usada como & Metodologia & $\begin{array}{l}\text { "Kozinets (1997), a netnografia pode ser utilizada de } \\
\text { três maneiras: a) como metodologia para estudar } \\
\text { ciberculturas e comunidades virtuais puras; b) como } \\
\text { metodologia para estudar ciberculturas e comunidades } \\
\text { virtuais derivadas; e c) como ferramenta exploratória } \\
\text { para estudar diversos assuntos. Para este autor as } \\
\text { comunidades virtuais puras são aquelas cujas relações } \\
\text { sociais se dão somente nas comunicações mediadas } \\
\text { pelo computador." }\end{array}$ \\
\hline Netnografia & $\begin{array}{l}\text { resumo de } \\
\text { textos verbais } \\
\text { na }\end{array}$ & Comunicação & $\begin{array}{l}\text { "A netnografia pode ser aplicada de forma mais rápida, } \\
\text { é menos dispendiosa, haja vista que se resume a } \\
\text { textos verbais, é menos subjetiva, pelo ato de ter } \\
\text { registros de vários tipos de materiais." }\end{array}$ \\
\hline Netnografia & $\begin{array}{l}\text { tem uma } \\
\text { abordagem }\end{array}$ & Etnografia & $\begin{array}{l}\text { "Netnografia cunhado na década de oitenta por Robert } \\
\text { V. Kozinets, como uma abordagem etnográfica para } \\
\text { pesquisas de marketing visando os estudos de } \\
\text { comportamentos de consumidores no ambiente virtual" }\end{array}$ \\
\hline
\end{tabular}

Apontamentos metodológicos iniciais sobre a netnografia no contexto pesquisa em comunicação digital e cibercultura

\begin{tabular}{|l|l|l|l|}
\hline Sujeito & Predicado & Objeto & Contextos de Citações \\
\hline Netnografia & $\begin{array}{l}\text { mapeamento } \\
\text { de perfis nas }\end{array}$ & $\begin{array}{l}\text { Comunidades } \\
\text { online }\end{array}$ & $\begin{array}{l}\text { "A netnografia também se apresenta como interessante } \\
\text { para o mapeamento dos perfis de consumo de seus } \\
\text { participantes a partir de suas práticas comunicacionais } \\
\text { nas plataformas sociais" }\end{array}$ \\
\hline
\end{tabular}

Inf. Inf., Londrina, v. 20, n. 1, p. 137 - 161, jan./abr. 2015. http:www.uel.br/revistas/informacao/ 


\begin{tabular}{|c|c|c|c|}
\hline Netnografia & $\begin{array}{l}\text { amplia o leque } \\
\text { epistemologis } \\
\text { mo da }\end{array}$ & Cibercultura & $\begin{array}{l}\text { "a netnografia como um dos métodos qualitativos que } \\
\text { amplia o leque epistemológico dos estudos em } \\
\text { comunicação e cibercultura, relacionando pesquisas } \\
\text { offline e online, de acordo com o tipo de } \\
\text { questionamento produzido em relação ao objeto em } \\
\text { sua coerência interna" (Orgad, 2009). }\end{array}$ \\
\hline Netnografia & $\begin{array}{l}\text { transposição } \\
\text { da etnografia } \\
\text { para formalizar } \\
\text { uma } \\
\text { complementar } \\
\text { outra }\end{array}$ & Metodologia & $\begin{array}{l}\text { "Kozinets (2007) recupera os quatro procedimentos } \\
\text { básicos de metodologia específicos da transposição da } \\
\text { etnografia para a netnografia. São elas: "Entrée } \\
\text { cultural; coleta e análise dos dados; ética de pesquisa; } \\
\text { e feedback e checagem de informações com os } \\
\text { membros do grupo" As etapas não acontecem de forma } \\
\text { estática, e os pesquisadores trabalham indo e vindo } \\
\text { por entre elas, apontando vivência de sobreposições e } \\
\text { interferências (aqui num sentido positivo) no qual os } \\
\text { procedimentos acontecem de forma interligada." } \\
\text { (Amaral, 2008). } \\
\text { "a netnografia, principalmente de forma complementar } \\
\text { a outros métodos", }\end{array}$ \\
\hline Netnografia & $\begin{array}{l}\text { valida no } \\
\text { campo da }\end{array}$ & Comunicação & $\begin{array}{l}\text { "Netnografia, ou etnografia virtual e sua adoção é } \\
\text { validada no campo da comunicação pelo fato de que } \\
\text { muitos objetos de estudo localizam-se no ciberespaço" } \\
\text { (Montardo \& Rocha, 2005: 01) e demandam } \\
\text { instrumental apropriado para sua análise." }\end{array}$ \\
\hline Netnografia & $\begin{array}{l}\text { Virtualização } \\
\text { da } \\
\text { premissas } \\
\text { básicas da }\end{array}$ & Etnografia & $\begin{array}{l}\text { "O termo netnografia tem sido amplamente utilizado } \\
\text { pelos pesquisadores da área do marketing e da } \\
\text { administração enquanto o termo etnografia virtual é } \\
\text { mais utilizado pelos pesquisadores da área da } \\
\text { antropologia e das ciências sociais. Para fins didáticos, } \\
\text { utilizamos ambos como sinônimos" } \\
\text { "A netnografia mantém as premissas básicas da } \\
\text { tradição etnográfica (Sá, 2002: 159) levantadas a partir } \\
\text { dos trabalhos de Geertz (2001): manter postura inicial } \\
\text { de estranhamento do pesquisador em relação ao } \\
\text { objeto; considerar a subjetividade; considerar os dados } \\
\text { resultantes como interpretações de segunda e terceira } \\
\text { mão; e finalmente considerar o relato etnográfico como } \\
\text { sendo de textualidades múltiplas." }\end{array}$ \\
\hline
\end{tabular}

Fonte: Elaborado pelas autoras.

A partir da extração conceitual e verificação de citações, elaborou-se a seguinte estrutura conceitual que serve de base para a nanopublicação. 
Figura 4 - Mapa conceitual de estruturação dos conceitos.

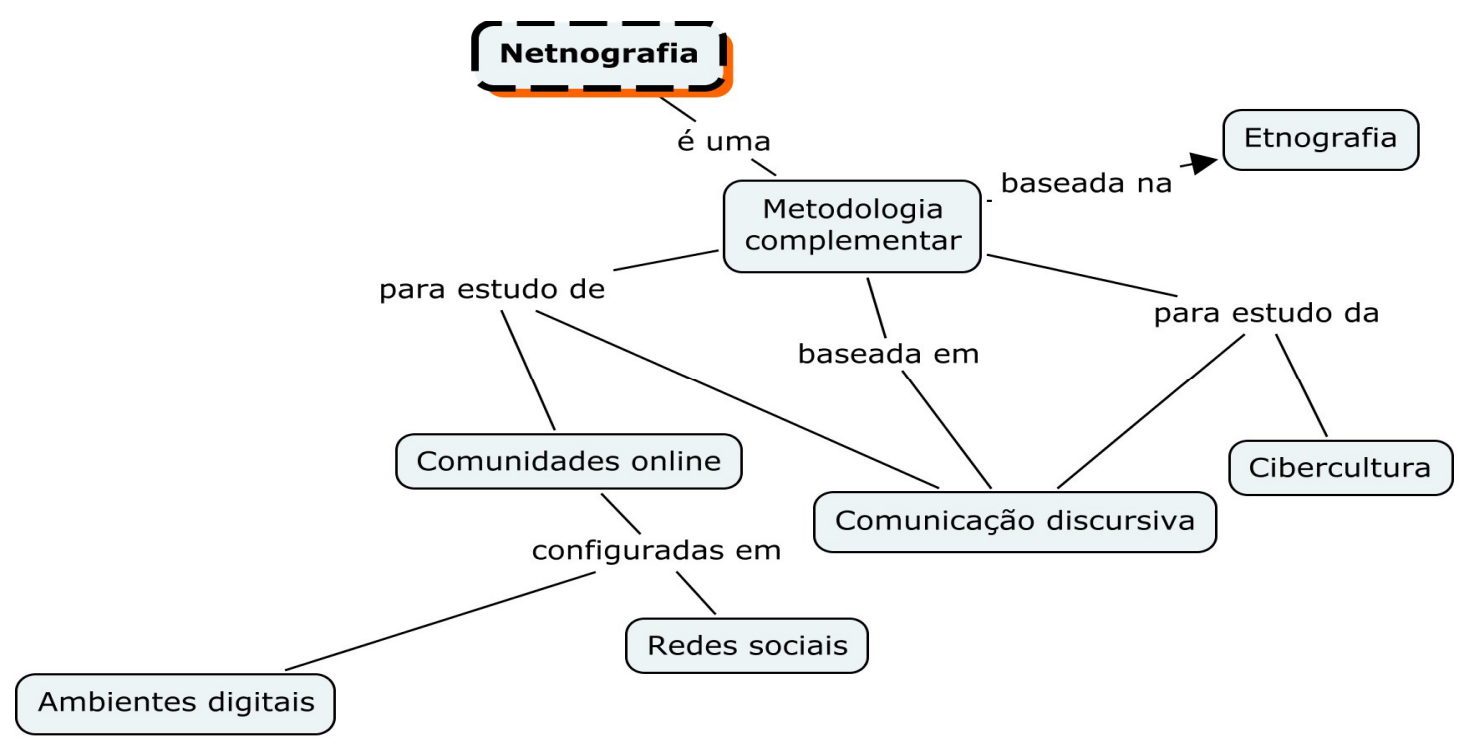

Fonte: Elaborado pelas autoras.

Observando a estrutura conceitual proposta na figura 4 verifica-se que a extração conceitual permite a construção de anotações que caracterizarão a definição de "Netnografia". Diante disso pode-se estabelecer a seguinte anotação: "a Netnografia é uma metodologia baseada nas técnicas da Etnografia utilizada para a complementação de processos metodológicos e para a análise de comunidades e contextos da cibercultura", que também está representada na figura 5 . 
Figura 5 - Nanopublicação sobre a Netnografia.

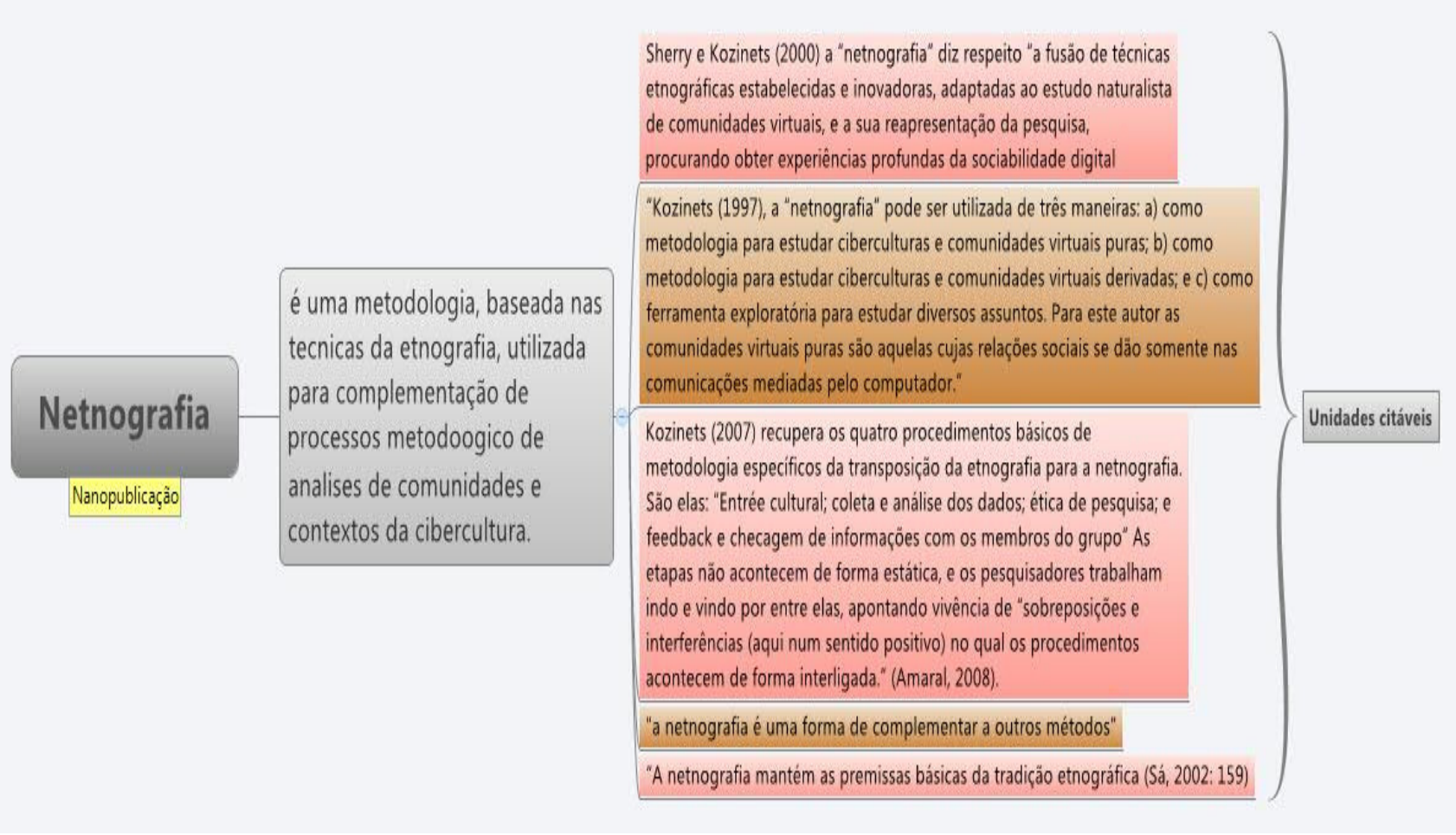

Fonte: Elaborado pelas autoras.

A figura 5 transmite a estruturação da nanopublicação composta pela anotação conceitual geral, seguida por quadrados subsequentes que são os desdobramentos com as declarações extraídas dos artigos analisados neste experimento. Observando as formações conceituais percebe-se nas e-evidencias as contribuições de comunidades e autorias citáveis para definir a "Netnografia". 
Tabela 4 - E-evidências

\begin{tabular}{|c|c|c|c|}
\hline \multirow[b]{2}{*}{ Artigo } & \multirow[b]{2}{*}{ Autoria } & \multicolumn{2}{|c|}{ Metadados de representação temática } \\
\hline & & $\begin{array}{l}\text { Referencias das Citações da } \\
\text { Nanopublicação }\end{array}$ & $\begin{array}{l}\text { Comuni } \\
\text { dades } \\
\text { de } \\
\text { conheci } \\
\text { mento }\end{array}$ \\
\hline $\begin{array}{l}\text { Estudo dos blogs } \\
\text { a partir da } \\
\text { netnografia: } \\
\text { possibilidades e } \\
\text { Limitações }\end{array}$ & Liliana Maria Passerino & $\begin{array}{l}\text { "Segundo Kozinets (1997), a netnografia } \\
\text { pode ser empregada de três formas: (1) } \\
\text { como ferramenta metodológica para } \\
\text { estudar comunidades virtuais puras; (2) } \\
\text { como ferramenta metodológica para } \\
\text { estudar comunidades virtuais derivadas; } \\
\text { e (3) como ferramenta exploratória para } \\
\text { diversos assuntos". }\end{array}$ & \\
\hline $\begin{array}{l}\text { Netnografia: } \\
\text { incursões } \\
\text { metodológicas na } \\
\text { cibercultura }\end{array}$ & $\begin{array}{l}\text { Paula Jung Rocha1 } \\
\text { Feevale } \\
\text { Sandra Portella } \\
\text { Montardo2 } \\
\text { Feevale }\end{array}$ & $\begin{array}{l}\text { Segundo Kozinets (2002) a netnografia é é } \\
\text { definida como um método de pesquisa } \\
\text { derivado da técnica etnográfica } \\
\text { desenvolvida no campo da antropologia } \\
\text { e, costuma-se dizer que a netnografia } \\
\text { tem conhecido um crescimento } \\
\text { considerado devido à complexidade das } \\
\text { experiências da sociedade digital. Este } \\
\text { método é constantemente utilizado por } \\
\text { pesquisadores das áreas da co }\end{array}$ & $\begin{array}{l}\text { Comunic } \\
\text { ação } \\
\text { Licenciat } \\
\text { ura em } \\
\text { Computa } \\
\text { ção }\end{array}$ \\
\hline $\begin{array}{l}\text { "Netnografia": } \\
\text { uma abordagem } \\
\text { para estudos de } \\
\text { usuários no } \\
\text { ciberespaço }\end{array}$ & $\begin{array}{l}\text { Virginia Bentes Pinto } \\
\text { Casemiro Silva Neto } \\
\text { Maria de Fátima Costa } \\
\text { Fabiola M. Pereira } \\
\text { Bezerra } \\
\text { Heliomar Cavati Sobrinho } \\
\text { Maria do R.Fatima P. } \\
\text { Cysne }\end{array}$ & $\begin{array}{l}\text { Kozinets (1997), a "netnografia" pode } \\
\text { ser utilizada de três maneiras: a) } \\
\text { como metodologia para estudar } \\
\text { ciberculturas e comunidades virtuais } \\
\text { puras; b) como metodologia para } \\
\text { estudar ciberculturas e comunidades } \\
\text { virtuais derivadas; e c) como } \\
\text { ferramenta exploratória para estudar } \\
\text { diversos assuntos. Para este autor as } \\
\text { comunidades virtuais puras são } \\
\text { aquelas cujas relações sociais se dão } \\
\text { somente nas comunicações mediadas } \\
\text { pelo computador." }\end{array}$ & $\begin{array}{l}\text { bliotecono } \\
\text { ia } \\
\text { iência da } \\
\text { formação } \\
\text { ecnologia }\end{array}$ \\
\hline $\begin{array}{l}\text { Apontamentos } \\
\text { metodológicos } \\
\text { iniciais sobre a } \\
\text { netnografia no } \\
\text { contexto } \\
\text { pesquisa em } \\
\text { comunicação } \\
\text { digital e } \\
\text { cibercultura. }\end{array}$ & $\begin{array}{l}\text { Adriana Amaral } \\
\text { Geórgia Natal } \\
\text { Lucina Viana }\end{array}$ & $\begin{array}{l}\text { Kozinets (2007) recupera os quatro } \\
\text { procedimentos básicos de metodologia } \\
\text { específicos da transposição da etnografia } \\
\text { para a netnografia. São elas: "Entrée } \\
\text { cultural; coleta e análise dos dados; ética } \\
\text { de pesquisa; e feedback e checagem de } \\
\text { informações com os membros do grupo" } \\
\text { As etapas não acontecem de forma } \\
\text { estática, e os pesquisadores trabalham } \\
\text { indo e vindo por entre elas, apontando } \\
\text { vivência de }\end{array}$ & $\begin{array}{l}\text { Comunic } \\
\text { ação }\end{array}$ \\
\hline
\end{tabular}

Fonte: Elaborado pelas autoras.

$\mathrm{Na}$ tabela 4 pode-se verificar que Kozinets é o autor de referência que define a "netnografia" uma vez que é uma citação presente em todos os artigos analisados. Também observa-se destaque do uso metodológico da "netnografia" nas 
comunidades ligadas ao domínio da Comunicação Social associada a Tecnologia. Por fim a seleção de metadados pôde ser estabelecida em dois campos: um que oriente a extração e divulgação das citações sobre "netnografia" e o outro na identificação das comunidades de conhecimento relacionadas aos autores da obra.

\section{ANÁLISE}

A elaboração da nanopublicação sobre "Netnografia" proporcionou a percepção de que a interlocução metodológica com os princípios de indexação não é só possível como eficiente. $O$ experimento limitou-se apenas pela representação de um elemento conceitual que foi a definição de "Netnografia". Esta decisão foi tomada devido ao excessivo trabalho de extração de fragmentos textuais relacionados aos outros conceitos destacados, como: "Comunidades Digitais", "Cibercultura", "Etnografia", etc. Pode-se intuir que os relacionamentos conceituais possíveis com as palavras referenciadas é um potencial que a automação do processo de construção da nanopublicação poderá evidenciar com maior robustez.

Deve se destacar que o experimento proposto neste artigo corroborou com o propósito da atribuição de crédito às citações, que identificaram e caracterizaram a nanopublicação, pois além da identificação das principais definições de "Netnografia" foi evidenciado o autor deste conceito, de sobrenome "Kozinete", o qual foi citado em todos os artigos analisados e mencionado como principal mentor dos estudos "Netnográficos".

Além disso, ficou expressa a necessidade de representação temática de dois elementos no corpo de metadados, segundo as e-evidências. $O$ primeiro está relacionado à citação direta da nanopublicação e o outro está orientado a identificação das comunidades de origem dos autores dos artigos.

\section{CONSIDERAÇÕES FINAIS}

A intensa adoção de recursos tecnólogicos nos processos de produção do conhecimento promove constantes inovações nos mecanismos de identificação, representação e disseminação de informações. A obsolescência tem se manifestado em segmentos que vão desde a elaboração de hardwares até os mecanismos (softwares) de produção e distribuição de conteúdos em meio digital. Neste contexto, 
a elaboração de nanopublicações faz-se eficiente para potencializar o compartilhamento de dados com a finalidade de gerar novos conhecimento.

Observa-se que a guarda e a comunicação de informações tende a sofrer alterações irreversíveis, quando se trata da satisfação das necessidades de informação de usuários especializados. Diante disso, torna-se imprescindível observar os contextos físicos, cognitivas e sociais nos quais as informações são elaboradas e disseminadas. A partir dessa observação pode-se estruturar mecanismos de representação que dêem conta de precisar um conteúdo informacional, sem distorções em relação ao contexto e ao entendimento conceitual. Neste aspecto, o experimento proposto neste artigo verificou que as nanopublicações podem ser consideradas promissoras, desde que seus aspectos teóricos e metodológicos sejam amplamente estudados, discutidos e disseminados.

A proposta de elaboração de uma nanopublicação seguiu alguns requisitos postulados pela CWA. Porém, as aplicações computacionais não foram contempladas. Além disso, o principal objetivo desse estudo consistia em evidenciar o caráter metodológico de união de alguns princípios da indexação com os postulados da nanopublicação.

Diante dos visíveis avanços do conhecimento e da consequente explosão informacional, deve-se buscar maneiras de impulsionar o uso da crescente produção documental, através de mecanismo de representação de informações coerentes com as demandas sócio/tecnológicas vigentes. Produzir conhecimento de maneira ágil, como normalmente é proposto pelos órgãos de fomento da ciência, pode exigir maneiras de recuperação de informação tão precisas quanto as unidades citáveis caracterizadas pelas nanopublicações.

\section{REFERÊNCIAS}

CESARINO, Maria Augusta da Nóbrega; PINTO, Maria Cristina Mello Ferreira.

Análise de assunto. Revista de Biblioteconomia de Brasília, Brasília, v. 8, n. 1, p. 32-43, jan./jun. 1985.

GROTH, Paul; GIBSON, Andrew; VELTEROP, Jan. The anatomy of a nanopublication. Journal Information Services and Use, Amsterdam, v. 30, n. 1-2, p. 51-56, Jan. 2010. 
. The open pharmaceutical triple store (Open PHACTS) projects begins.

2011. Disponível em: < http://nanopub.org/wordpress/?page_id=212>. Acesso em: 14 mar. 2014.

HJØRLAND, Birger. Information seeking and subject representation: activitytheoretical approach to information science. Michigan: Greenwood Press, 1997.

LANCASTER, Frederick Wilfrid. Indexação e resumo: teoria e prática. Brasília: Briquet de Lemos/Livros, 2004.

MOURA, Maria Aparecida. Informação, ferramentas ontologicas e redes sociais: a interoperabilidade na construção de tesauros ontologicos. In: CONGRESSO ISKO, 9., 2009, Valencia. Anais... Valencia, 2009.

Folksonomias, redes sociais e a formação para o tagging literacy: desafios para a organização da informação em ambientes colaborativos virtuais. Informação \& Informação, Londrina, v. 14, n. 1, p. 25-45, 2009.

MONS, Barend; VELTEROP, Jan. Nanopublication in the e-science era. 2009. Disponível em: <http://www.w3.org/wiki/images/4/4a/HCLS\$\$SWC2009 \$\$Workshop\$Mons.pdf>. Acesso em: 14 mar. 2014.

NANOPUB.org. Disponível em: <http://nanopub.org/wordpress/>. Acesso em: 14 mar. 2014.

CHICHESTER, Christine et al. The open PHACTS nanopublication guidelines.

Disponivel em: <http://www.nanopub.org/guidelines/1.8/>. Acesso em: 14 mar. 2014.

WILSON, Patrick. Two kinds of power. an essay on bibliographical control.

Berkeley: University of California Press, 1968.

Title

Nanopublications and indexation: processes and relationships

\section{Abstract}

Introduction: The study assumes that indexing and providing concepts in the primary source can offer subsidies to scientific sharing and collaboration in the construction of new knowledge. It presents the concept of nanopublication and establishes an interconnection between this emerging model of information representation in digital environments and guidelines postulated by the information indexing process.

Objective: It is presented the concept of nanopublication interfaced with indexing, under the organization of information processes in context. It was carried out a nanopublication modeling experiment that aimed to analyze the feasibility of elaborating an information organization methodology that allows the creation of an information representation model in virtual environments for the spread of networked knowledge.

Methodology: The methodology has been established in two stages. In the first stage, it was 
carried out a conceptual comparison between nanopublications and indexing. In the second stage, based on the guidelines of nanopublications, it is established the informational dimension of the method by generating the mapping of: the elements of the corpus, the conceptual extraction, the application of indexing principles, and the nanopublication modelling. Articles related to the concept of "netnography" were selected and analyzed from the selection made in "Google Scholar". The articles obtained from this process belonged to a common field of knowledge, the Applied Social Sciences, and to different domains such as: Administration, Communication Science, and Information Science.

Results: The preparation of nanopublication on "netnography" provided the perception that the methodological dialogue with the principles of indexing is possible and efficient. The proposed experiment in this paper corroborated the purpose of granting credit to quotes, identified and characterized in the nanopublication, because besides identifying the main definitions of "netnography", the author of this concept was evidenced, who was quoted in all analyzed articles and mentioned as the main reference in netnographic studies. Moreover, it was stated the need of thematic representation of two elements in the metadata corpus, according to the e-evidences. The first relates to the direct quote in nanopublications and the second is directed to the identification of the communities of origin of the authors of the articles.

Conclusions: It is considered that, given the visible advances in knowledge and the resulting information explosion in network, it should be sought ways to boost the use of increasing document production through information representation mechanism coherent with the current social/technological demands. Currently, producing knowledge in a fast way requires forms of information retrieval as accurate as the quotable units characterized by nanopublications.

Keywords: Nanopublication. Indexing. Information organization in context - methodology.

\section{Título}

Nanopublicaciones e indexación: procesos y relaciones

\section{Resumen}

Introducción: El estudio asume que indexar y disponibilizar conceptos en fuente primaria puede ofrecer subsidios para el compartir y para la colaboración científica en la construcción de nuevos conocimientos. Se presenta el concepto de nanopublicaciones (nanopublications) y se establece una interconexión entre este modelo emergente de la representación de la información en entornos digitales y las directrices postuladas por el proceso de indexación de información.

Objetivo: Se presenta el concepto de nanopublicaciones interconectado con la indexación, bajo la organización de los procesos de información en contexto. Hube un experimento de modelación de nanopublicaciones que tuvo como objetivo analizar la viabilidad de elaboración de una metodología de organización de la información para la creación de un modelo de representación de la información en entornos virtuales para la difusión del conocimiento en red.

Metodología: La metodología se ha establecido en dos etapas. En la primera etapa, se lleva a cabo la comparación conceptual entre la temática de las nanopublicaciones temáticas y la indexación. En la segunda etapa, con base en los lineamientos de la nanopublicación, se establece la dimensión informativa del método a través de la generación de mapeo de los 
elementos del corpus, de la extracción conceptual, de la aplicación de principios de indexación y del modelado de la nanopublicación. Fueron seleccionados y analizados los artículos relacionados con el concepto "netnografía" a partir de una selección realizada en el "Google Académico". Los artículos obtenidos pertenecían a un ámbito común de conocimientos, las Ciencias Sociales Aplicadas, y las diferentes áreas tales como: Administración, Comunicación y Ciencias de la Información.

Resultado: La preparación de la nanopublicación sobre "netnografía" proporcionó la percepción de que es posible y eficiente el diálogo metodológico con los principios de indexación. El experimento propuesto en este documento corroboró la concesión de crédito a las citas, identificadas y caracterizadas en la nanopublicación, porque además de la identificación de las principales definiciones de "netnografía", el autor de este concepto fue evidenciado, y él fue citado en todos los artículos analizados y mencionado como la principal referencia en los estudios netnográficos. Por otra parte, se expresó la necesidad de representación temática de dos elementos en el cuerpo de metadatos, segundo las eevidencias. El primer está relacionado a las citas directas en nanopublicaciones y el según está direccionado a la identificación de las comunidades de origen de los autores de los artículos.

Conclusiones: Se considera que, dados los avances visibles en el conocimiento y la consecuente explosión de la información en la red, se debe buscar maneras de aumentar el uso de la producción de documentos a través de mecanismo de representación de la información consistente con las demandas socio/tecnológicas. Actualmente, producir conocimiento de una manera rápida, requiere formas de recuperación de la información tan exactas cuanto las unidades citables caracterizadas por las nanopublicaciones.

Palabras clave: Nanopublicación. Indexación. Organización de la información en su contexto - metodología.

Recebido em: 15.05.2014

Aceito em: 13.06.2015 